\title{
Polarization switching of lithium niobate with giant internal field
}

\author{
L.-H. Peng, ${ }^{\text {a) }}$ Y.-C. Fang, and Y.-C. Lin \\ Institute of Electro-Optical Engineering, National Taiwan University, Taipei, Taiwan, Republic of China
}

(Received 8 September 1998; accepted for publication 11 February 1999)

\begin{abstract}
We report a high-field analysis on the sidewise $180^{\circ}$ domain motion in congruent-grown Z-cut lithium niobate $\left(\mathrm{LiNbO}_{3}\right)$ crystals. Polarization switching in reverse poling is found to take place at a smaller field strength $(E)$, exhibit a larger switching current $\left(i_{s}\right)$, and substantiate a faster switching time $\left(t_{s}\right)$ compared with the forward poling case. Such an axial anisotropy is correlated to a defect-induced internal field $E_{\text {int }}$ in the bulk and has a strength of $2.37 \mathrm{kV} / \mathrm{mm}$ and pointing along the crystal $c$ axis. In the high-field regime ranging from 19 to $24 \mathrm{kV} / \mathrm{mm}$, a linear dependence of $i_{s}$ and $1 / t_{s}$ on $E$ is resolved. These observations are ascribed to a fast nucleation process followed by a sidewise $180^{\circ}$ domain expansion, whose velocity can be characterized according to $\nu_{s}$ $=\mu_{s}\left[E-\left(E_{c} \pm E_{\text {int }}\right)\right]$, where the threshold field $E_{c}$ and mobility $\mu_{s}$ measure a strength of 18.74 $\mathrm{kV} / \mathrm{mm}$ and $1.6 \pm 0.1 \mathrm{~mm}^{2} / \mathrm{kV} \mathrm{s}$, respectively. (C) 1999 American Institute of Physics.
\end{abstract}

[S0003-6951(99)04914-1]

The abundance of novel mechanical, optical, and acoustical properties in ferroelectrics has made the study of this material system an important course in condensed matter physics $^{1}$ and quantum electronics. ${ }^{2}$ A plethora of research interest has been actively pursued quasi-phase-matching (QPM) (Ref. 3) of ferroelectric crystals in which the use of periodic $180^{\circ}$ domain reversal has found great potential in nonlinear frequency generation. Optical parametric infrared and short-wavelength harmonic generation in lithium niobate $\left(\mathrm{LiNbO}_{3}\right),{ }^{4}$ lithium tantalate $\left(\mathrm{LiTaO}_{3}\right),{ }^{5}$ and many other nonlinear crystals, ${ }^{6}$ represent one such exciting achievement. Although much attention has been focused on the applications of waveguide ${ }^{7}$ and bulk ${ }^{8}$ QPM devices such as periodically poled $\mathrm{LiNbO}_{3}$ (PPLN) and $\mathrm{LiTaO}_{3}$ (PPLT), little is known about the mechanism governing domain reversal in these crystals. Common practice has, therefore, adapted an empirical procedure of utilizing chemical diffusion followed by heat treatment in fabricating QPM devices. ${ }^{9}$ Albeit simple in its nature, employment of such methods genuinely invokes irregularities in the inverted domain boundaries ${ }^{10}$ and loss of optical nonlinearity ${ }^{11}$ that are known to be detrimental to the nonlinear conversion efficiency. ${ }^{12}$ It is, therefore, desirable to develop alternative domain inversion methods that are immune to these problems.

Recently, there has been a revival of interest in pursuing the pulsed field poling technique to achieve periodic domain reversal in nonlinear crystals such as $\mathrm{LiNbO}_{3}$ (Ref. 13) and $\mathrm{LiTaO}_{3}{ }^{14}$ In this method, a pulsed field with a strength $E$ greater than the coercive field of the corresponding crystal is required to initiate the polarization switching. ${ }^{15}$ Although pulsed field poling has been recognized as one of the most promising techniques in studying the dynamics of domain inversion in many of the ferroelectrics such as barrium titanate $\left(\mathrm{BaTiO}_{3}\right)$ and triglycine sulfate $(\mathrm{TGS}){ }^{1}$ by far, however, there have been limited successes in conceiving the kinetics of polarization switching in technologically important non-

\footnotetext{
${ }^{a)}$ Also with the Department of Electrical Engineering. Electronic mail: peng@cc.ee.ntu.edu.tw
}

linear crystals such as $\mathrm{LiNbO}_{3}$ (Ref. 16) and $\mathrm{LiTaO}_{3} \cdot{ }^{17}$

In this letter, we report a series of poling experiments on $Z$-cut congruent-grown $\mathrm{LiNbO}_{3}$ crystals in which we have used a transient current analysis to investigate the kinetics of polarization switching in $\mathrm{LiNbO}_{3}$ at various field strengths and poling directions. We thereby are able to observe a linear dependence of $i_{s}$ and $1 / t_{s}$ in the high-field regime, and resolve a unique axial anisotropy in these properties. The origins of these phenomena are ascribed to a field-driven sidewise $180^{\circ}$ domain motion whose velocity $\nu_{s}$ is characterized by $\nu_{s}=\mu_{s}\left[E-\left(E_{c} \pm E_{\text {int }}\right)\right]$, where $E_{c}$ and $E_{\text {int }}$ measure a threshold and internal field strength of 18.74 and 2.37 $\mathrm{kV} / \mathrm{mm}$, respectively, and $\mu_{s}$ a lateral mobility of 1.6 $\pm 0.1 \mathrm{~mm}^{2} / \mathrm{kV} \mathrm{s}$.

For high-field poling of $\mathrm{LiNbO}_{3}$, a high-voltage power supply made from IRCO (modified model C12K-20 for 12 $\mathrm{kV}$ output) or Glassman (model EH-15 for $15 \mathrm{kV}$ output) was used. The experimental setup was similar to that originally designed by Myers et al. ${ }^{18}$ in which a sealed Teflon fixture was electrically contacted to the $\mathrm{LiNbO}_{3}$ substrates via a saturated lithium chloride $(\mathrm{LiCl})$ aqueous solution. In order to eliminate the $\mathrm{RC}$ charging current ${ }^{19}$ and block the charge backflow ${ }^{20}$ at the beginning and termination of the poling process, we typically delivered pulses with a base-line field strength greater than $12 \mathrm{kV} / \mathrm{mm}$. By doing so, we can ensure a stabilized polarization switching process in $\mathrm{LiNbO}_{3} \cdot{ }^{17}$

The $500 \mu \mathrm{m}$ thick, double-sided polished, $\mathrm{Z}$-cut $\mathrm{LiNbO}_{3}$ substrates were purchased from Crystal Technology, with a congruent composition of $\mathrm{Li}_{2} \mathrm{O} /\left(\mathrm{Li}_{2} \mathrm{O}+\mathrm{Nb}_{2} \mathrm{O}_{5}\right)=0.484$. We follow the nomenclature of Ref. 17 in naming the starting crystals as the virgin crystals. The first polarization switching of the virgin crystals will be called forward poling and the second reversal back to its original state the reverse poling. A typical measurement of the switching current during a forward $(23 \mathrm{kV} / \mathrm{mm})$ and a reverse $(20 \mathrm{kV} / \mathrm{mm})$ poling process are shown in Figs. 1(a) and 1(b), respectively. We first note a "time lag" between the applied field and the subsequently occurring switching current. This phenomenon has been well 

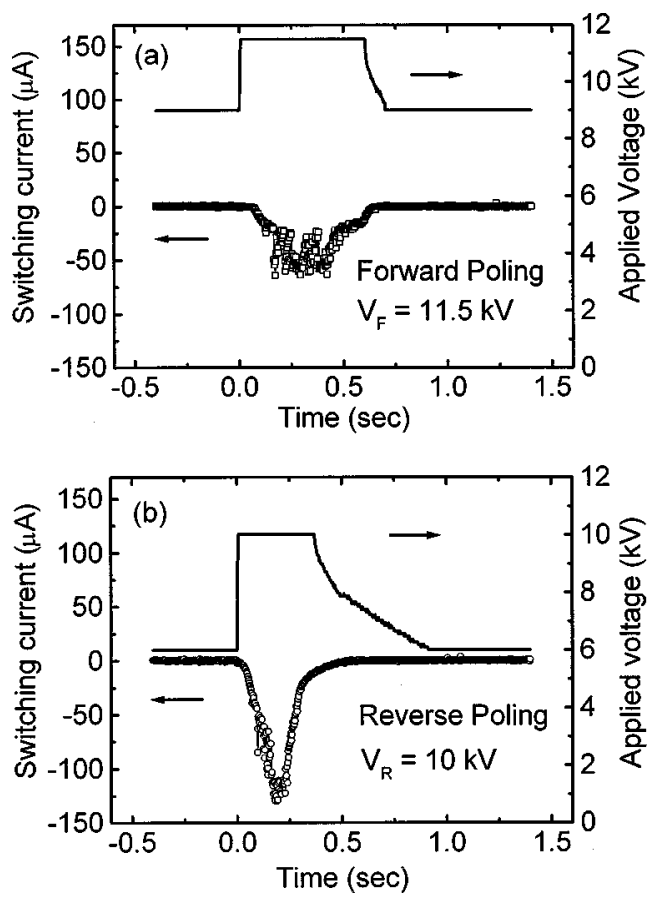

FIG. 1. Wave forms of the applied voltage and switching current during (a) forward poling at a field strength of $23 \mathrm{kV} / \mathrm{mm}$, and (b) reverse poling at 20 $\mathrm{kV} / \mathrm{mm}$ on a $0.5 \mathrm{~mm}$ thick $\mathrm{LiNbO}_{3}$ crystal.

known during the process of polarization switching in many ferroelectric crystals. ${ }^{21}$ The spikes residing in the current wave form of Fig. 1 correspond to Barkhausen pulses that signify the fusion and trapping of the domain motion by localized defects. ${ }^{22}$ A close examination of Fig. 1 also reveals the following axial anisotropy. Polarization switching in reverse poling can take place at a smaller field strength $(E)$, exhibit a larger switching current $\left(i_{s}\right)$, and substantiate a faster switching time $\left(t_{s}\right)$ compared with the forward poling case. Moreover, we have found as shown in Fig. 2, a linear dependence of $1 / t_{s}$ and $i_{s}$ on $E$; which, on the other hand, are independent of the poling direction. These observations are known to characterize a sidewise motion of the $180^{\circ}$ domain wall in the high-field regime. ${ }^{23}$ In comparison, polarization switching in the low-field regime is known to follow an exponential dependence on $E^{24}$

For crystals poled in the high-field regime, we have also found the switched domains on two sides of the etched $\mathrm{LiNbO}_{3}$ surfaces resemble the mirror images of each other as they would have been if the domain walls ran perpendicular to the crystal substrate. The cross-section optical micrograph

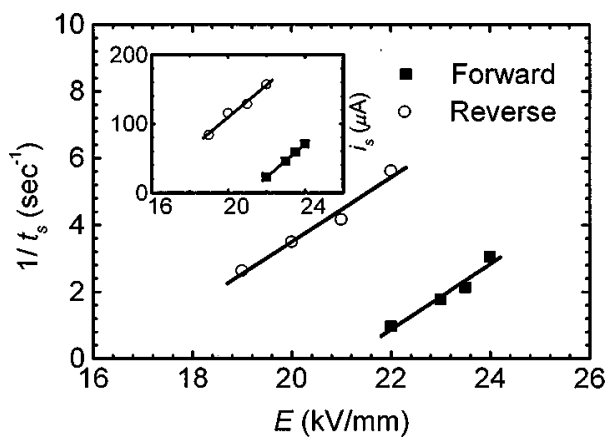

FIG. 2. Dependence of the switching time $\left(t_{s}\right)$ and switching current $\left(i_{s}\right)$ in high-field forward and reverse poling.

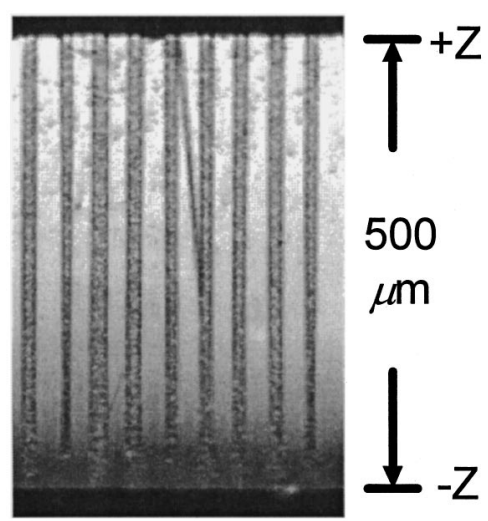

FIG. 3. Cross-sectional view of the $+y$ face of PPLN forward poled at 23 $\mathrm{kV} / \mathrm{mm}$.

of an etched $y$ face of PPLN, shown in Fig. 3, confirms such a fast and straight nucleation process along the crystal $c$ axis. In addition to revealing a good aspect ratio, this piece of PPLN contains approximately a 50\% duty cycle at a period of $40 \mu \mathrm{m}$ across the $500 \mu \mathrm{m}$ thick substrate, albeit it is poled at a field strength of $23 \mathrm{kV} / \mathrm{mm}$. We note the latter field strength is much higher than the commonly used value of $21.5 \mathrm{kV} / \mathrm{mm}$ known to maximize the domain nucleation density in $\mathrm{LiNbO}_{3} .{ }^{20}$

Combined with the information shown above, we are led to consider a nucleation type of mechanism as responsible for the polarization switching in congruent-grown $Z$-cut $\mathrm{LiNbO}_{3}$ crystals. In this model, fast nucleation occurs preferentially in the crystal $c$ axis along the existing $180^{\circ}$ domain boundary, and serves to propagate the boundary in a sidewise direction. ${ }^{25}$ The apparent sidewise velocity of the $180^{\circ}$ $\mathrm{LiNbO}_{3}$ domain motion in the high-field regime can, therefore, be characterized according to the following equation of motion:

$$
\nu_{s}=\mu_{s}\left[E-\left(E_{c} \pm E_{\text {int }}\right)\right] .
$$

Here, we have used an internal field strength $E_{\text {int }}$ to signify the axial anisotropy and $\mu_{s}$ the lateral mobility. Alternatively, $E_{c} \pm E_{\text {int }}$ in Eq. (1) represents a threshold field strength above which polarization switching can take place in forward (+) and reverse (-) poling, respectively.

In support of this model analysis, we replot in Fig. 4 the experimental $1 / t_{s}$ data in a reduced unit of $E^{\prime}=E-E_{c}$ with a $E_{c}$ value of $18.74 \mathrm{kV} / \mathrm{mm}$. Using Eq. (1), we have not only described a linear dependence of $1 / t_{s}$ on $E^{\prime}$ but also a deci-

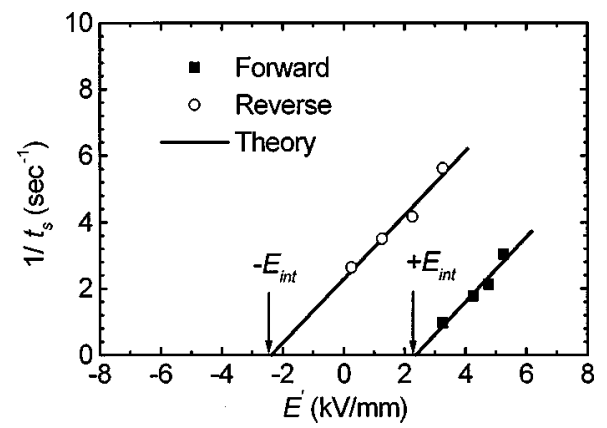

FIG. 4. Kinetic analysis of the lateral $180^{\circ}$ domain motion in the high-field regime using Eq. (1). Note the intersections with the abscissa at $\pm E_{\text {int }}$ $=2.37 \mathrm{kV} / \mathrm{mm}$. 


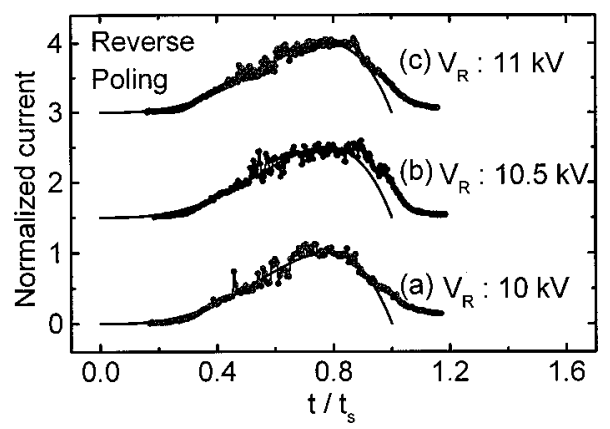

FIG. 5. Transient current analysis in the high-field reverse poling of $\mathrm{LiNbO}_{3}$ at (a) 20, (b) 21, and (c) $22 \mathrm{kV} / \mathrm{mm}$, respectively. Curves (b) and (c) have been shifted upwards for easy comparison.

sive internal field from the intersection with the abscissa at $\pm E_{\mathrm{int}}=2.37 \mathrm{kV} / \mathrm{mm}$. We have found a less than $10 \%$ variation in $E_{\text {int }}$ from wafer to wafer. Such a giant internal field is thought to originate from the nonstoichiometric point defects in the congruent-grown $\mathrm{LiNbO}_{3}$ crystals. ${ }^{26}$ We note the entry of an internal field $E_{\text {int }}$ in the analysis represents an important step toward understanding the kinetics of polarization switching in PPLN. For example, from the slope of $d\left(1 / t_{s}\right) / d\left(E^{\prime}\right)$ in Fig. 4 , one can deduce a constant lateral mobility $\mu_{s}$ of $1.6 \pm 0.1 \mathrm{~mm}^{2} / \mathrm{kV} \mathrm{s}$. This material property characterizes a $180^{\circ}$ domain motion in the high-field regime and casts no dependence on the poling direction at all.

The evolution of $i_{s}$ has been known to be due to an interaction between the switched $(r)$ and unswitched $(R)$ domain walls during the poling process. ${ }^{27}$ The line shape $g(t)$ of the high-field switching current can thereby be described according to $g(t)=C \times\left(r^{3} / R^{3}\right)\left(1-r^{2} / R^{2}\right)$, where $C$ is a normalization constant. Using Eq. (1), we show in Fig. 5 the excellent agreement between the calculated and the measured switching currents taken at reverse poling voltages of $10,10.5$, and $11 \mathrm{kV}$, separately. In particular, we note the slowly rising edge and fast falling tail of the transient current in Fig. 5 follow closely to a $t^{3}$ and $t^{5}$ dependence, respectively, on the elapsed poling time.

In summary, we report a high-field analysis on the kinetics of polarization switching in congruent-grown $Z$-cut $\mathrm{LiNbO}_{3}$ crystals. We observe an axial anisotropy in the switching current $\left(i_{s}\right)$ and the sweep rate $\left(1 / t_{s}\right)$ of the lateral $180^{\circ}$ domain motion on the poling direction. These observations are attributed to an internal field $E_{\text {int }}$ of $2.37 \mathrm{kV} / \mathrm{mm}$ associated with the nonstoichiometric point defects. This volume effect in turn modifies the sidewise $180^{\circ}$ domain motion according to $\nu_{s}=\mu_{s}\left[E-\left(E_{c} \pm E_{\text {int }}\right)\right]$ and results in a linear dependence of $i_{s}$ and the sweep rate $1 / t_{s}$ on $E$.

The authors wish to acknowledge discussions with Professor W. S. Wang and technical support from the Integrated Optics and Solid State Laboratories at N.T.U.E.E. This research was supported by the National Science Council under Grant Nos. 88-2112-M-002-032 and 87-2215-E-002-011.

${ }^{1}$ W. Känzik, in Solid State Physics, edited by F. Seitz and D. Turnbull (Academic, New York, 1957), Vol. 4.

${ }^{2}$ See, for example, IEEE J. Quantum Electron. 33, (1997); O. Auciello, J. F. Scott, and R. Ramesh, Phys. Today 51, 7 (1998).

${ }^{3}$ J. A. Armstrong, N. Bloembergen, J. Ducuing, and P. S. Pershan, Phys. Rev. 127, 1918 (1962).

${ }^{4}$ L. E. Myers, R. C. Eckardt, M. M. Fejer, R. L. Byer, and W. R. Bosenberg, Opt. Lett. 21, 591 (1996).

${ }^{5}$ K. Mizuuchi, K. Yamamota, and M. Kato, Appl. Phys. Lett. 70, 1201 (1997)

${ }^{6}$ L. M. Eng, H.-J. Güntherodt, G. Rosenman, A. Skliar, M. Oron, M. Katz, and D. Eger, J. Appl. Phys. 83, 5973 (1998).

${ }^{7}$ J. Webjörn, S. Siala, D. W. Nam, R. G. Waarts, and R. J. Lang, IEEE J. Quantum Electron. 33, 1673 (1997).

${ }^{8}$ L. E. Myers and W. R. Bosenberg, IEEE J. Quantum Electron. 33, 1663 (1997).

${ }^{9}$ L. Huang and N. A. F. Jaeger, Appl. Phys. Lett. 65, 1763 (1994).

${ }^{10} \mathrm{~J}$. Webjörn, F. Alurell, and G. Arvidsson, IEEE Photonics Technol. Lett. 1, 316 (1989).

${ }^{11}$ F. Laurell, M. G. Roelofts, and H. Hsiung, Appl. Phys. Lett. 60, 301 (1992).

${ }^{12}$ M. M. Fejer, G. A. Magel, D. H. Jundt, and R. L. Byer, IEEE J. Quantum Electron. 28, 2631 (1992).

${ }^{13}$ M. Yamada, N. Nada, M. Saitoh, and K. Watanabe, Appl. Phys. Lett. 62, 435 (1993).

${ }^{14}$ K. Mizuuchi and K. Yamamoto, Appl. Phys. Lett. 66, 2943 (1995).

${ }^{15}$ I. Camlibel, J. Appl. Phys. 40, 1690 (1969).

${ }^{16}$ V. Gopalan and M. C. Gupta, Ferroelectrics 198, 49 (1997).

${ }^{17}$ V. Gopalan and T. E. Mitchell, J. Appl. Phys. 83, 941 (1998).

${ }^{18}$ L. E. Myers, R. C. Eckardt, M. M. Fejer, R. L. Byer, W. R. Bosenberg, and J. W. Pierce, J. Opt. Soc. Am. B 12, 2102 (1995).

${ }^{19}$ A. Kuroda, S. Kurimura, and Y. Uesu, Appl. Phys. Lett. 69, 1565 (1996).

${ }^{20}$ G. D. Miller, R. G. Batchko, W. M. Tulloch, D. R. Weise, M. M. Fejer, and R. L. Byer, Opt. Lett. 22, 1834 (1997).

${ }^{21}$ R. C. Miller and A. Savage, Phys. Rev. 112, 755 (1958).

${ }^{22}$ A. G. Chynoweth, Phys. Rev. 110, 1316 (1958).

${ }^{23}$ H. L. Stadler and P. J. Zachmanidis, J. Appl. Phys. 34, 3255 (1963).

${ }^{24}$ W. J. Mertz, Phys. Rev. 95, 690 (1954).

${ }^{25}$ Ferrodelectrics and Related Materials, edited by G. A. Smolenskii (Gordon and Breach Science, New York, 1984), Chap. 7.

${ }^{26}$ V. Gopalan, T. E. Mitchell, Y. Furukawa, and K. Kitamura, Appl. Phys. Lett. 72, 1981 (1998).

${ }^{27}$ E. Fatuzzo, Phys. Rev. 127, 1999 (1962). 